\title{
A novel technique based on Bloch surface waves sustained by one-dimensional photonic crystals to probe fluid dynamics in a microfluidic channel
}

\author{
Agostino Occhicone ${ }^{1, *}$, Alberto Sinibaldi, ${ }^{1}$ Frank Sonntag ${ }^{2}$, Peter Munzert $^{3}$, Norbert Danz, ${ }^{3}$ \\ and Francesco Michelotti ${ }^{1}$ \\ 1. SAPIENZA Università di Roma, Department of Basic and Applied Sciences for Engineering, Via A. \\ Scarpa, 16, 00161 Roma, Italy \\ 2. Fraunhofer Institute for Material and Beam Technology IWS, Winterbergstr. 28, 01277 Dresden, \\ Germany \\ 3. Fraunhofer Institute for Applied Optics and Precision Engineering, A.-Einstein-Str. 7, 07745 Jena, \\ Germany
}

\begin{abstract}
We report on the use of an optical sensing platform based on Bloch surface waves sustained by onedimensional photonic crystals as a novel optical tool to probe in real time the fluid flow at a boundary wall of a microfluidic channel under dynamic conditions. Understanding how fluid flow interacts with wall surfaces is crucial for a broad range of biological processes and engineering applications, such as surface wave biosensing. The proposed platform provides nanometric resolution with respect to the distance from the boundary wall. Here, for the first time, we report on the investigation on the temporal evolution of the interface between two fluids with different refractive indices under convective and diffusive conditions. The temporal evolution of the fluids interface in proximity of the wall is retrieved from the experimental measurements. From the data analysis, the diffusion coefficients of glucose and glycerol in water are evaluated and found in good agreement with the literature. Tuning the one-dimensional photonic crystals geometry and the Bloch surface waves' dispersion has the potential to probe the fluid flow in an extremely wide range of distances from the microfluidic channel wall.
\end{abstract}

Keywords: $\mu$-fluidics, Sensors, one-dimensional Photonic Crystals, Bloch Surface Waves.

\footnotetext{
${ }^{*}$ Corresponding author

Email: agostino.occhicone@uniroma1.it

$\dagger$ Electronic Supplementary Information (ESI) available

See DOI: $10.1039 / \mathrm{x} 0 \mathrm{x} x 00000 \mathrm{x}$
} 


\section{INTRODUCTION}

During the past decade, a wide range of protein microarray technologies have been developed promoting rapid progress in the fields of experimental and clinical investigations [1,2].

In particular, microfluidics and the design of micro-total analysis systems ( $\mu$-TAS) are the most promising aspects to address the inherent complexity of biological systems with massive experimental parallelization and measurements of protein-protein, cell-protein and inter cellular interactions in real-time [3-5]. Extending from pure microfluidics to more fully developed and automated lab-on-chip (LoC), entire sample preparation protocols can be embedded preceding the biological assay, such as fluid transport, fluid metering, fluid mixing, and separation or concentration of molecule [6]. In this framework, microfluidics is uniquely devoted at manipulating liquids in networks of micro-channels providing increased resolution of the analysis and sampling throughput, while reducing the cost per assay. On the other hand, at such reduced volumes, fluids exhibit different physical properties, when compared to their behaviour at the macro-scale [7]. As stated, since at the micro/nano - scale the fluid behaviour can be extremely different, phenomena as surface tension, heat conduction, and fluidic resistance start to play a crucial role.

However, particle transport within fluids can be appropriately treated by convective and diffusion equation (CDE), that comes from a combination of the diffusion equations, Fick's law, with the hydrodynamic Navier-Stokes equations (NSE) [6,8-10]. This covers two different transport mechanisms: the first is the molecular diffusion of the solute mainly driven by local concentration gradients into the solution, the second is the convective fluid motion, and concerns the hydrodynamic transport of solute in a given solvent along the channel $[6,9,10]$. The solution of those equations provides temporal evolution and spatial distribution of solute concentration $\mathrm{c}(\mathrm{x}, \mathrm{y}, \mathrm{z}, \mathrm{t})$, and the velocity profiles that characterize the fluid flow.

In addition, micro-environmental conditions, such as tumour surrounding tissues/fluids, are extremely difficult to mimic due to their intrinsic complexity and hard to model using conventional approaches. Microfluidic platforms could address many of these limitations and recreate more closely the physiological microenvironment that is normally experienced by cells/tissues in the human body [7].

In this work we propose a new integrated optical approach to effectively monitor the fluid's dynamic features and exploiting the peculiar properties of Bloch surface waves (BSW) propagating at the truncation edge of a one dimensional photonic crystal (1DPC). The use of other types of surface waves, namely surface plasmon polaritons (SPP) [11], was proposed by Loureiro et al. [9].

1DPC are dielectric multi-stacks that, under specific conditions, can sustain a BSW at the interface between the external liquid environment (the fluid under investigation) and the photonic structure itself [12,13]. The penetration depth of the evanescent tail of the BSW can be varied, at a given operation wavelength, by choosing the thicknesses and the refractive indices of the dielectric materials constituting the 1DPC layers. Such evanescent field can be used as a nanoscopic probe to locally investigate the properties of fluids [14]. The main goal of the present work is to make use of a phase-sensitive sensor based on BSW to evaluate the diffusion characteristics of glucose aqueous solutions under dynamic flow conditions in the proximity of the 
1DPC surface. For the particular 1DPC reported here, the intensity of the evanescent tail of the BSW decreases within the fluid with a decay length, $d$, that is of the order of $120 \mathrm{~nm}$; this means that the system is able to detect refractive index variations in a small laminar volume very close to the sensor surface. By injecting into a microfluidic channel solutions with different solute concentrations, that means different refractive indices, one can follow in real time the changes of the BSW features for a variety of different experimental conditions. From such investigations, we can address the transport of solutes in aqueous solutions in both convective and diffusive regimes.

The proposed approach could meet an important need in microfluidics technologies, which create devices useful in fields from medical testing such as drug delivery to toxin detection. It could be particularly important for miniaturized devices that rely on volume measurements to report, for example, the concentration of a particular molecule in a mixture or characterize in terms of diffusion of such molecules in a biological environment.

\section{OPTOFLUIDIC DETECTION TECHNIQUE}

\subsection{DPC optical transducer}

The confinement of BSW at the interface between a finite 1DPC and an external dielectric medium is guaranteed by Bragg reflection and total internal reflection (TIR) conditions on the two sides of the interface, respectively $[12,13,15]$. The excitation of BSW at a given wavelength $\lambda_{0}$ is obtained using a prism coupler in the so-called Kretschmann-Raether TIR configuration [12,13,15], as revealed by the appearance of a dip at an angle $\theta_{\text {RES }}$ in the angular reflectance. A perturbation of the external refractive index in proximity of the 1DPC surface can be observed and quantified by tracking the angular position of such a dip.

The 1DPCs utilised in this work were fabricated by plasma ion assisted electron beam evaporation under high vacuum conditions on standard microscope slides. The dielectric materials are $\mathrm{SiO}_{2}$ (silica), $\mathrm{Ta}_{2} \mathrm{O}_{5}$ (tantala) and $\mathrm{TiO}_{2}$ (titania). The deposition rates were: $0.5 \mathrm{~nm} / \mathrm{s}$ for $\mathrm{SiO}_{2}, 0.4 \mathrm{~nm} / \mathrm{s}$ for $\mathrm{Ta}_{2} \mathrm{O}_{5}$ and $0.25 \mathrm{~nm} / \mathrm{s}$ for the $\mathrm{TiO}_{2}$ layer [14].

The geometry of the 1DPC is shown in Figure 1b: starting from the substrate, there is a first silica matching layer, a periodic part with two tantala/silica bilayers and a topping thin titania/silica bilayer. The thicknesses are $\mathrm{d}_{\mathrm{SiO} 2}=275 \mathrm{~nm}, \mathrm{~d}_{\mathrm{Ta} 2 \mathrm{O} 5}=120 \mathrm{~nm}$ for the periodic part and $\mathrm{d}_{\mathrm{TiO} 2}=20 \mathrm{~nm}, \mathrm{~d}_{\mathrm{SiO} 2}=20 \mathrm{~nm}$ for the topping layers. The refractive indices of the layers are: $\left(\mathrm{TiO}_{2}\right) 2.280+1.8 \mathrm{E}-3 \mathrm{i},\left(\mathrm{SiO}_{2}\right) 1.474+5 \mathrm{E}-6 \mathrm{i},\left(\mathrm{Ta}_{2} \mathrm{O}_{5}\right)$ $2.106+5 \mathrm{E}-5 \mathrm{i}$ at $\lambda_{0}=670 \mathrm{~nm}$. The 1DPC was designed to sustain a TE polarized BSW at $\lambda_{0}$ when operating with an aqueous external fluid $\left(\mathrm{n}_{\mathrm{EL}}=1.330\right)$ and optimized to show the maximum sensitivity to refractive index perturbations:

$$
\mathrm{S}=\frac{\partial \theta_{\mathrm{RES}}}{\partial \mathrm{n}_{\mathrm{EL}}}
$$


For the present 1DPC, the sensitivity was measured experimentally by means of solutions with calibrated refractive indices and found to be $\mathrm{S}=34 \mathrm{deg} / \mathrm{RIU}$ (where RIU is "refractive index unit").

Numerical simulations of the reflectance were carried out by means of the transfer matrix method (TMM) [15] to calculate the electromagnetic field in the 1DPC. In Figure 1c we show the calculated reflectance in either deionized (DI) water or a 5\% wt solution of glucose in DI water, showing the resulting shift of the resonance $\operatorname{dip}(\Delta \theta=0.25 \mathrm{deg})$. One should note that the resonance is much sharper $(0.135 \mathrm{deg})$ than the resonance found for equivalent SPR sensors operating under the same TIR conditions (about $1.7 \mathrm{deg}$ ).

In Figure 1d we show the calculated BSW intensity profile in the 1DPC when the sensor is excited at resonance. The intensity is plotted as a function of the optical distance from the sensor surface normalized to the operation wavelength $\mathrm{y}_{\mathrm{opt}} / \lambda_{0}$. The BSW decay length in the external medium d (geometric), where the intensity is attenuated by a factor 1/e with respect to value at the 1DPC surface, is found from the condition $\mathrm{y}_{\mathrm{opt}} / \lambda_{0}=0.224$ (see dashed lines in Figure 1d) and is $\mathrm{d}=0.224 \times \lambda_{0} / \mathrm{n}_{\mathrm{FL}}=113 \mathrm{~nm}$.

Figure $1 \mathrm{~d}$ shows a very important feature of BSW; if one scales the wavelength $\lambda$ and the optical thicknesses of the 1DPC layers by the same factor the plot does not change. This allows to tune the decay length $\mathrm{d}=0.224 \times \lambda / \mathrm{n}_{\mathrm{FL}}(\lambda)$ and probe the external fluid in a thinner/thicker layer close to the sensor surface. As examples, for the starting design at $\lambda_{0}$ given above, one would get $\mathrm{d}=67 \mathrm{~nm}$ for $\lambda=405 \mathrm{~nm}$ (InGaN laser) and $\mathrm{d}=263 \mathrm{~nm}$ for $\lambda=1550 \mathrm{~nm}$ (InGaAsP laser). Of course, such feature relies on the availability of transparent dielectrics for the 1DPC layers at the scaled wavelength, which is a relatively simple condition to satisfy. An even larger range of variation of $d$ can be achieved if one completely changes the 1DPC geometry, not only scaling it, even keeping $\lambda_{0}$ fixed [16].

\subsection{Optical setup}

The optical set-up is sketched in Figure 2. The light source is a temperature stabilized pigtailed laser diode (Thorlabs LPS-675-FC) with nominal wavelength $\lambda_{0}=670 \mathrm{~nm}$ at room temperature (RT). The pigtail's output is collimated and injected into the optical path by means of a dielectric mirror. The laser diode temperature and the current are stabilized by means of a laser diode controller (Profile LDC402B/TED 420). As described in $[12,13,15]$, an input polarizer (POL) sets the beam polarization at 45 deg with respect to the incidence plane, giving rise to TE and TM components with the same intensity. After reflection from the prism coupler, the beam passes through an analyzer (AN) that is crossed at $-45 \mathrm{deg}$. The role of the liquid crystal phase retarder cell (LCR) is to change the phase $\Psi$ between the TE and TM components and bias the input field to any state of elliptical polarization $[16,17]$. Such an ellipsometric configuration permits one to obtain very sharp resonant dips in the reflectance, even for 1DPC with very low losses [15].

Before reaching the prism coupler the beam is expanded by means of a telescope constituted by a microscope objective $(\mathrm{MO}, 40 \times)$ and a spherical lens $\left(\mathrm{SL}, \mathrm{f}_{1}=250 \mathrm{~mm}\right)$. After reducing the diameter to $1 \mathrm{~cm}$ by means of a diaphragm (DIA), the beam is focused on the sample by a cylindrical lens (CL1, $\mathrm{f}_{2}=150 \mathrm{~mm}$ ) through a 
BK7 coupling prism (Figure 2a). In the focal point of the telescope, a rotating diffuser (RD) is used in order to destroy the spatial coherence of the beam. A $\theta-2 \theta$ rotation stage allows one to set the average incidence angle $(\theta)$ around the resonance angular position when operating at $\lambda_{0}$ The reflected beam is collected at $2 \theta$ by a second cylindrical lens (CL2, $\mathrm{f}_{3}=150 \mathrm{~mm}$ ) (Fourier lens) and sent to a CMOS array detector (Thorlabs DCC1645C, 1280x 1024 pixel, 8 bit). The cylindrical focusing and Fourier imaging lenses allow to illuminate a sharp line at the biosensor surface perpendicularly to the incidence plane and to sense refractive index changes simultaneously and in parallel along such a line [18].

The 1DPC biochip back facet is contacted to the prism by means of a matching oil.

\subsection{Microfluidic cell and fluids handling system}

The 1DCP is topped with a polydimethylsiloxane (PDMS) fluidic cell that is defining a microfluidic channel in contact with the 1DPC surface [19]. The fluidic cell was cast from a laser micro patterned master and is tightly connected to an aluminum plate that can be temperature controlled by means of either a thermal bath (circulation of water with controlled temperature) or a Peltier element. Through the temperature controlled aluminum back plate we can assure a stable temperature of the sensors $\left( \pm 0.01{ }^{\circ} \mathrm{C}\right)$. As sketched in Figure 3, the fluidic channel has a $2 \mathrm{~mm}$ wide inlet and outlet, a central part of the channel that is $12.6 \mathrm{~mm}$ long and $4 \mathrm{~mm}$ wide, and two connection tapers (see figure S.1 in the supplementary material for the details of the cell geometry). The height of the fluidic channel is $120 \mu \mathrm{m}$. The surface of the channel is about $1.59 \mathrm{~cm}^{2}$ with a volume of $19 \mu \mathrm{L}$. During the measurements, the temperature of solutions was kept constant $\left(\mathrm{T}=26^{\circ} \mathrm{C}\right)$ by means a temperature controller (Julabo).

The fluids handling system has been developed with the aim to drive/move the fluids across the microfluidic channel. It consists of a motorized syringe $(1 \mathrm{~mL})$ pump (Cavro) and of a 10 positions stream selector (VICI Valco) allowing to pick solutions from several different cuvettes, as sketched in Figure 3. The connections were made out of PEEK tubings ( $250 \mu \mathrm{m}$ inner diameter). The syringe pump is used to suck the solutions from the drain end of the fluidic cell via a 4-way port that can be commuted to empty the syringe itself in the waste.

\section{EXPERIMENTAL RESULTS}

In Figure 3 (top), we show some CMOS images acquired when the fluidic channel is filled with DI water under steady state conditions. The images were acquired for three different positions of the illumination line along the fluidic channel: across the inlet, the intermediate region, and the central section of the channel. In the CMOS images, each row corresponds to a position along the illumination line. Along each row, the reflected intensity shows the resonant behaviour given in Figure 1c. Inside the fluidic channel the resonance is narrow and more or less in the middle of the CMOS image. In the region outside the channel, where the PDMS comes in touch with the 1DPC surface, the resonance is wider and shifted to the left hand side (larger angles), due to the increased refractive index. One should keep in mind that in the CMOS images $\theta$ increases 
from right to left. The broadening of the resonance is due to the increased losses in the PDMS region. In each image, the height corresponds to a $4 \mathrm{~mm}$ distance and the width corresponds to a 1 deg angular range.

The BSW sensors were used to study in real time the kinetics of the refractive index changes in proximity of the surface of the 1DPC, upon injection of glucose solutions in DI water with different concentrations. With reference to Figure 4, the exponential tail of the BSW evanescent field probes the refractive index changes averaged over an extended layer in proximity of the 1DPC surface, in the presence of an interface profile between the incoming solution and that previously filling the channel.

The experimental tests were carried out with flow rates $\Phi$ ranging from $1.353 \mu \mathrm{L} / \mathrm{s}$ to $31.4 \mu \mathrm{L} / \mathrm{s}$ and using DI water and glucose solutions in DI water. The tests were repeated several times and for transitions from DI water to glucose solutions, and vice versa. The images were acquired through the CMOS camera detector at a frame rate of 10 frame/s (the full videos are available in the supplementary material section).

As examples, in Figure 5 we show the CMOS images recorded at fixed time intervals for $\Phi=20.95 \mu \mathrm{L} / \mathrm{s}$, $10.52 \mu \mathrm{L} / \mathrm{s}$ and $2.699 \mu \mathrm{L} / \mathrm{s}$; the optical system was aligned to monitor the refractive index changes along a line right at the middle of the fluidic channel ( $4 \mathrm{~mm}$ wide). The sequences refer to the transition from a solution of $5 \%$ wt glucose in DI water to DI water.

In Figure 5a, in the first frame we show the profile of the reflected intensity along one of the CMOS camera rows in DI water (row 512). A sharp dip is observed at $\theta_{0}$, whose angular position is taken as a reference for the following frames, being determined by the refractive index of pure DI water. In the last frame of Figure 5a the images obtained at the beginning and at the end of the injection are superimposed so as to show the total shift of the BSW resonance along the illumination line due to the refractive index change at the surface. The acquired videos were analysed frame by frame and row by row. For each row, corresponding to a physical point $\mathrm{z}$ along the illumination line across the fluidic channel, we evaluated the angular shift $\Delta \theta(\mathrm{z}, \mathrm{t})=\theta(\mathrm{z}, \mathrm{t})-\theta_{0}$ of the BSW resonance as a function of time. The CMOS angular range is $1 \mathrm{deg}$ and is sampled with 1280 pixel, therefore the pixel/angle conversion factor is $7.813 \times 10^{-3} \mathrm{deg} /$ pixel. The BSW resonance shift is then converted to an effective index change by making use of the sensitivity defined above $\Delta \mathrm{n}_{\text {eff }}(\mathrm{z}, \mathrm{t})=\Delta \theta(\mathrm{z}, \mathrm{t}) / \mathrm{S}$.

As an example in Figure 6 we show the temporal dependence of the effective refractive index for a single CMOS camera row (row 512) corresponding to the point $\mathrm{z}=2 \mathrm{~mm}$ along the illumination line, i.e. at the centre of the fluidic channel. Every curve is taken at a different flow rate. As expected, there is a transition regime whose duration $\tau$ depends on the nominal flow rate $\Phi$. Here we define $\tau$ as the time needed to pass from $10 \%$ to $90 \%$ of the total $\Delta \mathrm{n}_{\text {eff }}$ change. As it can be seen, $\tau$ increases from seconds to hundreds of seconds when $\Phi$ decreases. The total effective refractive index change when the fluidic channel transits between the two stationary conditions in which it is filled either with the 5\% wt glucose solution or with DI water, is found to be $\Delta \mathrm{n}_{\text {eff }}(\infty)=-7.35 \times 10^{-3}$ RIU. Such a value is in very good agreement with that calculated 
through the linear empiric formula $\Delta \mathrm{n}_{\mathrm{MAX}}=\beta \cdot \mathrm{c}$ that relates the glucose concentration $\mathrm{c}$ to the increase of the solution refractive index with respect to pure DI water, with $\beta=1.47 \times 10^{-3} \mathrm{RIU} / \%$ at $\lambda_{0}[20]$.

\section{DISCUSSION}

The data acquired in the micro-opto-fluidic experiments with glucose solutions can be analysed in order to extract information about the transport and diffusion phenomena taking place in the volume probed by the BSW close to the 1DPC surface. It is therefore necessary to model the fluid flow inside the channel.

The general dynamic vector form of the differential convective diffusion equation follows from the continuity equation:

$$
\frac{\partial c}{\partial t}=-\nabla \cdot j=\mathcal{D} \nabla^{2} \mathbf{c}-\mathbf{v} \cdot \nabla \mathbf{c}
$$

where $\mathrm{j}$ is the mass flow and describes the motion of a ionic or macromolecular solute particle in a solvent stream, $\mathcal{D}$ is the solute diffusion coefficient and $\mathbf{v}$ the temporal and spatial velocity profile of the solution $[9,21]$. This equation is valid in the absence of sources and sinks, since the terms describing the desorption and adsorption of the solute are missing.

Tracking the temporal concentration variation and evaluating the transition time $\tau$ could be a good method to determine $\mathcal{D}$ through the analytical solutions of the Eq. (2) $[9,21]$.

In the asymptotic limit $\mathrm{v} \rightarrow 0$, at finite $\mathrm{c}$, the Eq. (2) reduces to the molecular diffusion equation:

$$
\frac{\partial c}{\partial t}=\mathcal{D} \nabla^{2} \mathbf{c}
$$

Analogously, in the limit $\mathrm{c} \rightarrow 0$, from the Eq. (2) we get the first Navier-Stokes equation (NSE), in which c is replaced by the solvent density $\rho$ :

$$
\frac{\partial \rho}{\partial t}=-\nabla(\rho v)
$$

that can be used to calculate the velocity profile under a pressure gradient $[21,22]$. The evaluation of the term $\partial \mathrm{c} / \partial \mathrm{t} \sim \Delta \mathrm{c} / \tau$, in the two limiting cases $\mathrm{v} \rightarrow 0$ and $\mathrm{c} \rightarrow 0$, can provide information on the diffusive and convective flow properties in a channel [9]. The Poiseuille's equation represents a simple analytical solution of the Eq. (4) for cylindrical and rectangular channels with low aspect ratio [21]. The ideal velocity profile across the channel section is parabolic, as sketched in Figure 4, with velocity at the walls equal to zero under the no-slip condition [10,21]. The validity of the no-slip condition is an open and controversial issue. In particular, the debate is open on the wall characteristic and if the condition is valid for solvo-phobic surfaces. Three kinds of experiments were performed to clarify this issue and some tentative conclusions were drawn: (a) no-slip conditions apply for almost all experiments with fluids that wet boundaries (e.g., Sharp et al. 2001; see, however, Pit et al. 2000 and Choi et al. 2002);

(b) a small amount of roughness on an otherwise slip surface produces an effective no-slip boundary condition (Richardson 1973, Jansons 1988, Zhu \& Granick 2002); 
(c) some experiments with partially wetting fluids produce results consistent with a slip boundary condition, possibly because of gas or vapor cavities or films along the boundary; this effect, and also adhesion, may be purposefully enhanced by a "spiky" roughness at small scales (e.g., Kim \& Kim 2002, Cottin-Bizonne et al. 2003).

In conclusion, except for special circumstances requiring care and clever design, the no-slip boundary conditions remain an excellent approximation for flows at scales above tens of nanometres [10].

From the Eq. (2) it is expected that the time derivative of the effective concentration, i.e. the concentration averaged in the laminar volume probed by the surface wave, is proportional to the flow rate $\Phi$, as also found in ref. [9], and given by:

$$
\frac{\mathrm{dc}_{\mathrm{eff}}}{\mathrm{dt}} \sim \frac{\Delta \mathrm{c}}{\tau}=\mathbf{m} \boldsymbol{\Phi}+\mathrm{v}_{\mathbf{0}}
$$

where $\mathrm{v}_{0}$ is the value in the limit $\Phi \rightarrow 0$, when the convective flow is absent, and can be related to the diffusive transit time $\tau_{\text {diff }}$ under a step concentration variation $\Delta \mathrm{c}$ by the relation:

$$
\mathbf{v}_{\mathbf{0}}=\frac{\Delta \mathbf{c}}{\tau_{\text {diff }}}
$$

From the Eqs.(5-6) one can derive the following dependency of the transit time on the flow rate:

$$
\tau=\frac{\tau_{\text {diff }}}{1+\gamma \Phi}
$$

predicting that for $\Phi \rightarrow 0$ the transit time is limited by the diffusion time.

In Figure 7 we plot the experimental values of $\tau$ that were extracted from the measurements shown in Figure 5 as a function of $\Phi$. For each experiment carried out at $\Phi$, we analysed the row by row CMOS images and extracted for each row the $\Delta \mathrm{n}_{\text {eff }}(\mathrm{z}, \mathrm{t})$ change across the channel as a function of time, as shown in the insets of Figure 7 for the exemplary case $\Phi=20.95 \mu \mathrm{L} / \mathrm{s}$. The $\tau$ values plotted in Figure 7 were obtained by averaging the values $\tau(\mathrm{z})$ found in the central region of the channel, $\mathrm{z} \in(0.8 \mathrm{~mm}, 3.2 \mathrm{~mm})$, in order to rule out the effects taking place at the side walls of the channel. Multiple experimental points shown in Figure 7a for each $\Phi$ correspond to data taken in repeated experiments, with transition from 5\% wt glucose solutions to DI water and vice versa.

The data in Figure $7 \mathrm{a}$, related to experiments carried out with glucose solutions, were interpolated (solid line) with the Eq. (7) and the value for $\tau_{\text {diff }}=(80 \pm 9) \mathrm{s}$ was found from the fit. One can then evaluate the diffusion coefficient in DI water as:

$$
\mathcal{D}=\frac{\mathbf{L}^{2}}{\boldsymbol{\tau}_{\text {diff }}}
$$

where we assumed the characteristic length of the fluidic system to be twice the fluidic cell height, i.e. $\mathrm{L}=2 \mathrm{~h}=0.240 \mathrm{~mm}[21]$. The value found for $\mathcal{D}_{\text {glu }}=(7.2 \pm 0.8) \times 10^{-10} \mathrm{~m}^{2} / \mathrm{s}$ is consistent with that reported in literature $6.7 \times 10^{-10} \mathrm{~m}^{2} / \mathrm{s}$ at $25^{\circ} \mathrm{C}[20]$. 
The same experiments were carried out with glycerol solutions in DI water and the experimental results are shown in Figure $7 \mathrm{~b}$. From the fit we find $\tau_{\text {diff }}=(65 \pm 6) \mathrm{s}$ and $\mathcal{D}_{\text {gly }}=(8.9 \pm 0.8) \times 10^{-10} \mathrm{~m}^{2} / \mathrm{s}$ is again in agreement with the literature value $1.0 \times 10^{-9} \mathrm{~m}^{2} / \mathrm{s}$ at $25^{\circ} \mathrm{C}[20]$.

The experiments can be further analysed to extract information on the time dependence of the distance $\xi(\mathrm{z}, \mathrm{t})$ of the interface between the solutions from the 1DPC surface (see Figure 4), for each point $\mathrm{z}$ of the illumination line. With reference to Figure 1d, the BSW evanescent tail is characterized by an intensity given by:

$$
|E|^{2}=A e^{-\frac{y}{d}}
$$

and probes the refractive index in an extended layer during the injection and the transit of the interface between the solutions. The variation of the effective refractive index $\Delta \mathrm{n}_{\text {eff }}(\mathrm{z}, \mathrm{t})$ recorded by the BSW sensor along the illumination line can be related to the difference between the refractive indices of the two solutions $\Delta \mathrm{n}$ and to $\xi(\mathrm{z}, \mathrm{t})$. Assuming that diffusion can be neglected $\Delta \mathrm{n}_{\mathrm{eff}}$ is:

$$
\Delta \mathbf{n}_{\text {eff }}(\mathbf{z}, \mathbf{t})=\mathbf{n}_{\text {eff }}(\mathbf{z}, \mathbf{t})-\mathbf{n}_{\mathbf{0}}=\frac{\int_{0}^{\xi(z, t)} \mathbf{n}_{\mathbf{0}} \mathbf{e}^{-\frac{y}{d} d y}+\int_{\xi(z, t)}^{\infty} \mathbf{n}_{\mathbf{s}} \mathbf{e}^{-\frac{y}{d}} d y}{\int_{0}^{\infty} e^{-\frac{y}{d}} d y}-n_{0}=\Delta \mathbf{n}_{\text {MAX }} e^{-\frac{\xi(z, t)}{d}}
$$

where $n_{0}$ and $n_{s}$ are the refractive indices of pure DI water and of the solution, respectively. From the Eq. (10) we get:

$$
\xi(\mathbf{z}, \mathbf{t})=\mathbf{d} \cdot \ln \frac{\Delta \mathbf{n}_{\mathrm{MAX}}}{\Delta \mathbf{n}_{\mathrm{eff}}(\mathbf{z}, \mathbf{t})}
$$

From the $\Delta \mathrm{n}_{\text {eff }}(\mathrm{z}, \mathrm{t})$ values, extracted from the images shown in Figure 5 and used to generate the $\tau$ plotted in Figure 7, we retrieved by means of the Eq. (6) the values of $\xi(\mathrm{z}, \mathrm{t})$. In Figure 8 we show the reconstructed maps of $\xi(\mathrm{z}, \mathrm{t})$, as a function of time and of the position across the fluidic channel, for $\Phi=20.95 \mu \mathrm{L} / \mathrm{s}$, $10.52 \mu \mathrm{L} / \mathrm{s}, 3.59 \mu \mathrm{L} / \mathrm{s}$, and $2.699 \mu \mathrm{L} / \mathrm{s}$. The maps shown in Figure 8 permit to put into evidence the temporal evolution of the interface during the injection. As expected, close to the side walls the change of $\xi(\mathrm{z}, \mathrm{t})$ is delayed with respect to the central portion of the channel. As it can be seen instabilities of the front can be observed. From the experimental value of the resolution of the sensor with respect to refractive index changes $\Delta_{\text {neff min }}=3 \times 10^{-6}$ RIU [13] we can determine the dynamic range for the measurement of $\xi(\mathrm{z}, \mathrm{t})$ and find have $\xi_{\max }=920 \mathrm{~nm}$ and $\xi_{\min }=0.1 \mathrm{~nm}$.

If diffusion cannot be neglected then the Eq. (11) is an underestimation of the real distance $\xi_{\text {diff }}(\mathrm{z}, \mathrm{t})$, due to the exponential feature of the BSW probe. This makes that the accuracy on the values of $\xi_{\text {diff }}(\mathrm{z}, \mathrm{t})$ is determined by diffusion and not by the noise of the measurement technique. In particular this is true for low values of $\Phi$ and in the limit $\xi(\mathrm{z}, \mathrm{t}) \rightarrow 0$.

The results shown in Figure 8 are in good qualitative agreement with numerical simulations of the instantaneous concentration distribution across the cell width, averaged in the probed depth. The simulations 
were carried out by means of the finite elements method (FEM) applied to the Eq. (2) and taking into account both convection and diffusion, for the microfluidic channel used here (see supplementary material).

\section{CONCLUSIONS}

In this work, we successfully studied the diffusion characteristics under dynamic flow conditions in presence of a 1DPC. By making use of a phase sensitive sensor based on BSW, we were able to estimate the diffusion coefficients in DI water for glucose and glycerol, respectively. Our results are in very good agreement with data found in literature. In addition, we extracted information on the time dependence of the distance $\xi(\mathrm{z}, \mathrm{t})$ of the interface between the solutions and the 1DPC surface.

Such novel approach permits to build a 3D interface surface between two fluid phases with nanometric resolution.

The immediate throughput for miniaturized devices is related to the possibility of measuring in real-time diffusion coefficients of protein species directly on $\mu$-TAS in clinical environment.

\section{ACKNOWLEDGEMENTS}

This work was funded by the European Commission through the project BILOBA (Grant Agreement 318035).

\section{REFERENCES}

[1] H. Zhu, M. Bilgin, R. Bangham, D. Hall, A. Casamayor, P. Bertone, N. Lan, R. Jansen, S. Bidlingmaier, T. Houfek, T. Mitchell, P. Miller, R. A. Dean, M. Gerstein, M. Snyder, Global Analysis of Protein Activities Using Proteome Chips, Science 293 (2001) 2101-2105. doi: 10.1126/science.1062191.

[2] R. B. Jones, A. Gordus, J. A. Krall and G. MacBeath, A quantitative protein interaction network for the ErbB receptors using protein microarrays, Nature 439 (2006), 168-174. doi: 10.1038/nature04177.

[3] T. M. Squires, R. J. Messinger and S. R. Manalis, Making it stick: convection, reaction and diffusion in surface-based biosensors, Nature 26 (2008), 417-426. doi: 10.1038/nbt1388.

[4] G. M. Whitesides, The origins and the future of microfluidics, Nature 442 (2006), 368-373. doi: 10.1038/nature05058.

[5] J. P. Lafleur, A. Jönsson, S. Senkbeil, J. P. Kutter, Recent advances in lab-on-chip for biosensing applications, Elsevier, Biosensors \& Bioelectronics $76 \quad$ (2016), 213-233. doi: 10.1016/j.bios.2015.08.003

[6] D. Psaltis, S. R. Quake and C. Yang, Developing optofluidic technology through the fusion of microfluidics and optics, Nature 442 (2006), 381-386. doi: 10.1038/nature05060. 
[7] D. Wlodkowic, J. M. Cooper, Tumors on chips: oncology meets microfluidics, Elsevier, Curr Opin Chem Biol. 14 (2010), 556-567. doi: 10.1016/j.cbpa.2010.08.016.

[8] T. M. Squires, S. R. Quake, Microfluidics: Fluid physics at the nanoliter scale, Reviews of Modern Physics 77 (2005), 977-1026. doi: 10.1103/RevModPhys.77.977.

[9] C. C. L. Loureiro, A. M. N. Lima, H. Neff, Optical monitoring of micro fluidic flow conditions, employing surface plasmon resonance sensing, Journal of Physics: conference series 85 (2007). doi: 10.1088/1742-6596/85/1/012023.

[10] H. A. Stone, A. D. Stroock and A. Ajdaris, Engineering Flows in Small Devices: Microfluidics Toward a Lab-on-a-Chip, Annual Review Fluid. Mech. 36 (2004), 381-411. doi: 10.1146/annurev.fluid.36.050802.122124.

[11] J. Homola, S. S. Yee and G. Gauglitz, Surface plasmon resonance sensors: review, Sens. Act. B 54 (1999), 3-15. doi: 10.1016/S0925-4005(98)00321-9

[12] A. Sinibaldi, A. Anopchenko, R. Rizzo, N. Danz, P. Munzert, P. Rivolo, F. Frascella, S. Ricciardi, F. Michelotti, Angularly resolved ellipsometric optical biosensing by means of Bloch surface waves, Analytical and Bioanalytical Chemistry 407 (2015), p. 3965-3974. doi: 10.1007/s00216-015-8591-8.

[13] A. Sinibaldi, N. Danz, A. Anopchenko, P. Munzert, S. Schmieder, R. Chandrawati, R. Rizzo, S. Rana, F. Sonntag, A. Occhicone, L. Napione, S. De Panfilis, M. M. Stevens, F. Michelotti, Label-Free Detection of Tumor Angiogenesis Biomarker Angiopoietin 2 Using Bloch Surface Waves on One Dimensional Photonic Crystals, Journal of Lightwave Technology 33 (2015), 3385-3393. doi: 10.1109/JLT.2015.2448795.

[14] A. Anopchenko, A. Occhicone, R.Rizzo, A. Sinibaldi, G. Figliozzi, N. Danz, P. Munzert and F. Michelotti, Effect of thickness disorder on the performance of photonic crystal surface wave sensors, Optics Express 24 (2016), 7728-7742. doi: 10.1364/OE.24.007728.

[15] A. Sinibaldi, R. Rizzo, G. Figliozzi, E. Descrovi, N. Danz, P. Munzert, A. Anopchenko and F. Michelotti, A full ellipsometric approach to optical sensing with Bloch surface waves on photonic crystals, Optics Express 21 (2013), 23331-23344. doi: 10.1364/OE.21.023331.

[16] R. Rizzo,N. Danz,F. Michelotti, E. Maillart, A. Anopchenko, C. Wächter, Optimization of angularly resolved Bloch surface wave biosensors, Optics Express 22 (2014), 23202-23214. doi: 10.1364/OE.22.023202.

[17] A. Vargas, R. Donoso, M. Ramírez, J. Carrión, M. del Mar Sánchez-López and I. Moreno, Liquid Crystal Retarder Spectral Retardance Characterization Based on a Cauchy Dispersion Relation and a Voltage Transfer Function, Opt. Rev. 20 (2013), 378-384. doi: 10.1007/s10043-013-0068-4.

[18] N. Danz, A. Kick, F. Sonntag, S. Schmieder, B. Höfer, U. Klotzbach, M. Mertig, Surface plasmon resonance platform technology for multi-parameter analyses on polymer chips, Eng. Life Sci. 11 
(2011), 566-572. doi: 10.1002/elsc.201000192.

[19] J. C. McDonald, D. C. Duffy, J. R. Anderson, D. T. Chiu, H. Wu, O. J. Schueller, G. M. Whitesides., Fabrication of microfluidic systems in poly(dimethylsiloxane), Electrophoresis 21 (2000), 27-40. doi: 10.1002/(SICI)1522-2683(20000101)21:1<27::AID-ELPS27>3.0.CO;2-C.

[20] Edited by D. R. Lide,. B. Raton, CRC handbook of chemistry and physics, 89th edition, CRC Press, Taylor and Francis Group (2009), 2736.

[21] B. J. Kirby, Micro- and Nanoscale Fluid Mechanics: Transport in Microfluidic Devices, Published in the United States of America by Cambridge University Press (2010).

[22] A. Kozachok, Navier -Stokes First Exact Transformation, Universal Journal of Applied Mathematics 1 (2013), 157-159. doi: 10.13189/ujam.2013.010301. 


\section{CAPTIONS FOR FIGURES}

Figure 1: (a) Sketch of the BSW excitation scheme making use of a prism coupler in the KretschmannRaether configuration and operating under TIR conditions. (b) Sketch of the 1DPC sensor (thicknesses not to scale). (c) TMM calculated reflectance for DI water and 5\%wt glucose solutions. (d) BSW transverse intensity profile calculated at resonant excitation in DI water; the intensity is plotted as a function of the optical distance from the 1DPC surface normalized to $\lambda_{0}$.

Figure 2: Optical set-up for the excitation of BSW on 1DPC in the Kretschmann-Raether TIR configuration. (LD) laser diode $\lambda_{0}=670 \mathrm{~nm}$, (POL input polarizer, (LCR) liquid crystal phase retarder, (MO) 40x microscope objective, $(\mathrm{RD})$ rotating diffuser, (SL) spherical lens $\mathrm{f}_{1}=250 \mathrm{~mm}$, (DIA) diaphragm, (CL1) and (CL2) cylindrical lenses with $\mathrm{f}_{2}=\mathrm{f}_{3}=150 \mathrm{~mm}$, (AN) output analyser, (CMOS) camera array sensor.

Figure 3: Sketch of the fluids handling system. A photograph of the PDMS cell with tubings is shown in the central inset. At the top we show the CMOS images acquired for different positions of the illumination line along the fluidic channel: across the inlet channel (grey) that is $2 \mathrm{~mm}$ wide, across the inlet channel smothering (blue), and across in the middle part of the channel (red) where the width is maximum (4 mm). The detailed description of the cell is given in Figure S.1.

Figure 4: Qualitative representation of the parabolic interface between a solution and the DI water injected in the microfluidic cell. The BSW probes the effective refractive index at the 1DPC surface.

Figure 5: Images extracted from the videos acquired by the CMOS camera when DI water is injected in a channel already filled with a 5\% wt glucose solution in Di water. The images shown here were collected at (a) $\Phi=20.95 \mu \mathrm{L} / \mathrm{s}$, (b) $\Phi=10.52 \mu \mathrm{L} / \mathrm{s}$, and (c) $\Phi=2.699 \mu \mathrm{L} / \mathrm{s}$. In the first frame of (a) we show the profile of the reflected intensity along one of the CMOS camera rows in DI water (row 512). In the last frame of (a) the images obtained at the beginning and at the end of the injection are superimposed to put into e evidence the resonance shift.

Figure 6: Measured temporal dependence of the effective refractive index change $\Delta \mathrm{n}_{\text {eff }}(\mathrm{t})$ for several different experimental values of $\Phi$. The curves were extracted from measurements similar to those reported in Figure 5 and correspond to the row 512 of the CMOS frames (centre of the channel). The method used to define $\tau$ is shown for the case $\Phi=1.353 \mu \mathrm{L} / \mathrm{s}$. 
Figure 7: Experimental values of $\tau$ as function of $\Phi$. For a given $\Phi, \tau$ was obtained from the $\Delta \mathrm{n}_{\text {eff }}(\mathrm{z}, \mathrm{t})$ decay surfaces shown in the insets and is the average of the values found across the channel for $\mathrm{z} \in(0.8 \mathrm{~mm}, 3.2$ $\mathrm{mm}$ ) Experiments carried out with 5\% wt solutions in DI water of: (a) glucose (b) glycerol. The red solid line is the interpolation with the Eq. (7) and the dashed line is the linear approximation valid for large $\Phi$ values. The insets show the $\Delta \mathrm{n}_{\text {eff }}(\mathrm{z}, \mathrm{t})$ change across the channel as a function of time in the exemplary case $\Phi=20.95 \mu \mathrm{L} / \mathrm{s}$.

Figure 8: Reconstructed experimental maps of $\xi(\mathrm{z}, \mathrm{t})$, as a function of time and of the position across the fluidic channel, for $\Phi=20.95 \mu \mathrm{L} / \mathrm{s}, 10.52 \mu \mathrm{L} / \mathrm{s}, 3.59 \mu \mathrm{L} / \mathrm{s}$, and $2.699 \mu \mathrm{L} / \mathrm{s}$. The maps put in evidence the temporal evolution of the interface during the injection of DI water when the fluidic cell is filled with a solution 5\% wt glucose in water. The white and black areas correspond to regions where $\xi(\mathrm{z}, \mathrm{t})$ could not be evaluated because the $\xi(\mathrm{z}, \mathrm{t})$ values are larger than the upper limit of the dynamic range of the sensor. 

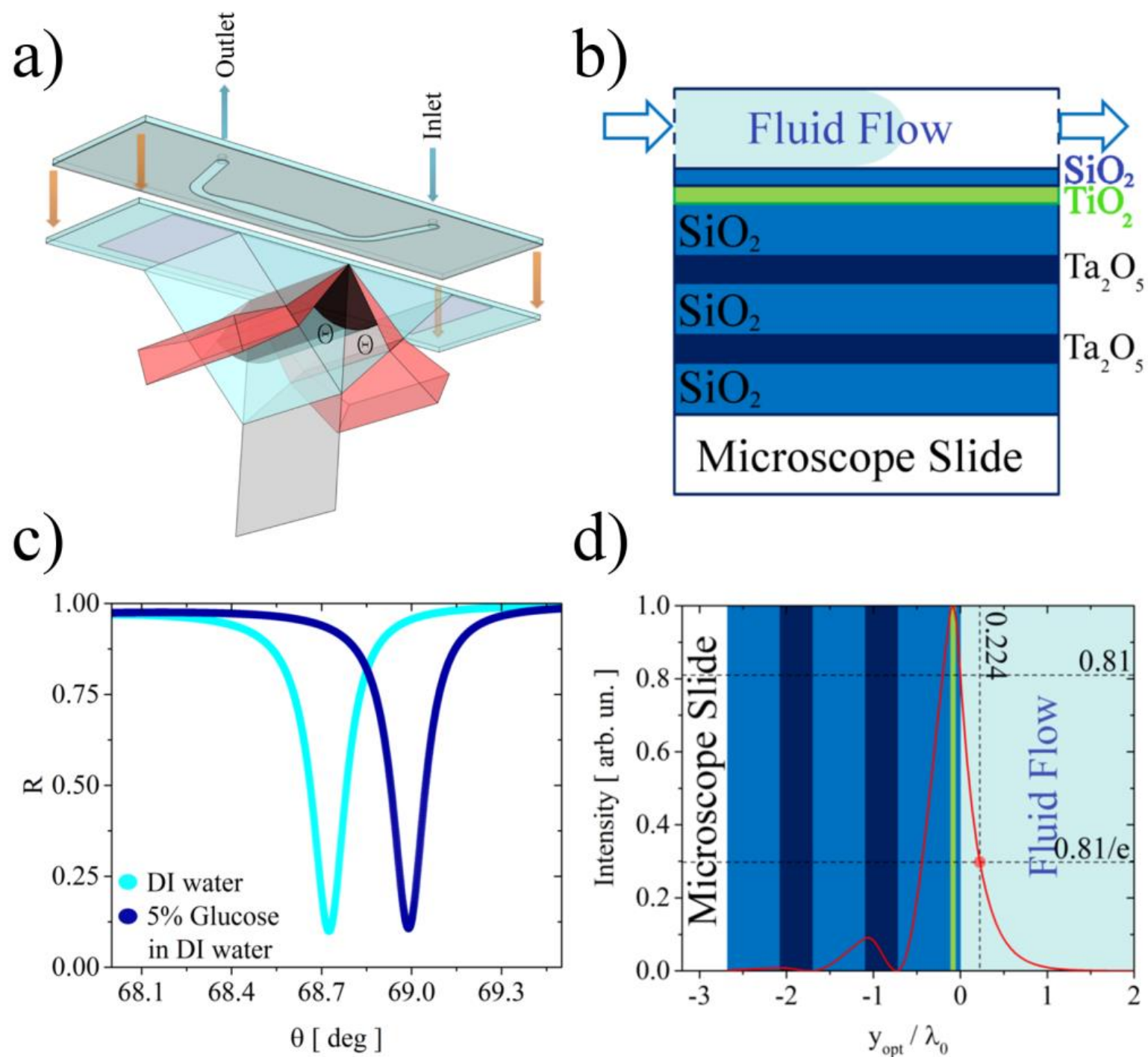

d)

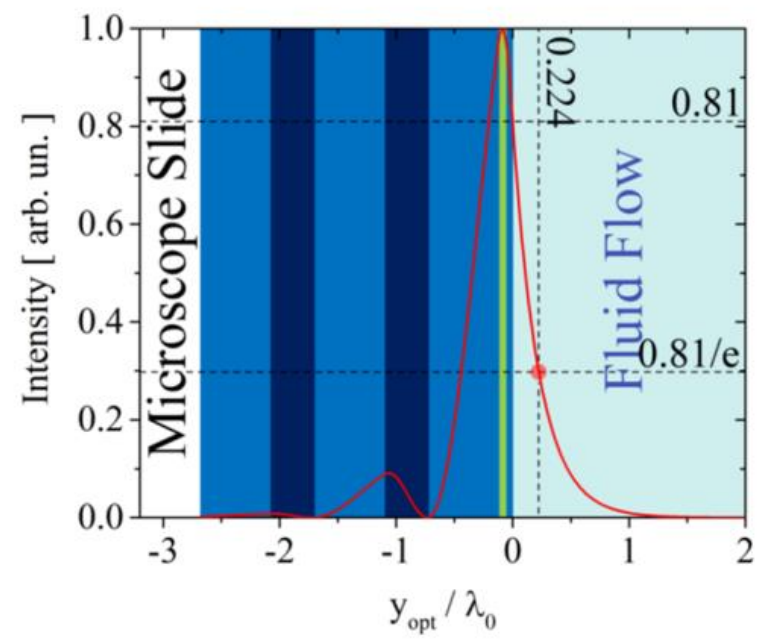

Figure 1: (a) Sketch of the BSW excitation scheme making use of a prism coupler in the KretschmannRaether configuration and operating under TIR conditions. (b) Sketch of the 1DPC sensor (thicknesses not to scale). (c) TMM calculated reflectance for DI water and 5\%wt glucose solutions. (d) BSW transverse intensity profile calculated at resonant excitation in DI water; the intensity is plotted as a function of the optical distance from the 1DPC surface normalized to $\lambda_{0}$.

A. Occhicone et al., Real-time microfluidic monitoring by means of Bloch surface waves at the surface of onedimensional photonic crystals, submitted to Journal of Sensors and Actuators B. 


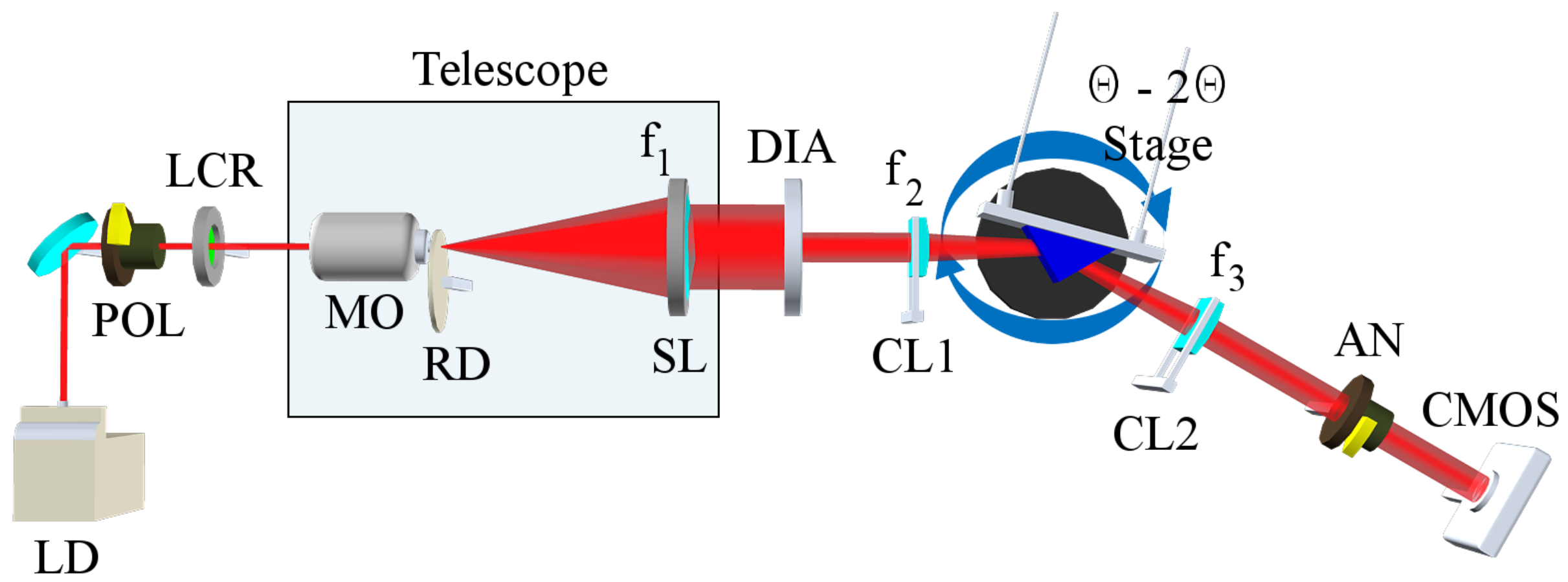

Figure 2: Optical set-up for the excitation of BSW on 1DPC in the Kretschmann-Raether TIR configuration. (LD) laser diode $\lambda_{0}=670 \mathrm{~nm}$, (POL input polarizer, (LCR) liquid crystal phase retarder, (MO) 40x microscope objective, (RD) rotating diffuser, (SL) spherical lens $\mathrm{f}_{1}=250 \mathrm{~mm},(\mathrm{DIA})$ diaphragm, (CL1) and (CL2) cylindrical lenses with $\mathrm{f}_{2}=\mathrm{f}_{3}=150 \mathrm{~mm}$, (AN) output analyser, (CMOS) camera array sensor.

A. Occhicone et al., Real-time microfluidic monitoring by means of Bloch surface waves at the surface of one-dimensional photonic crystals, submitted to Journal of Sensors and Actuators B. 


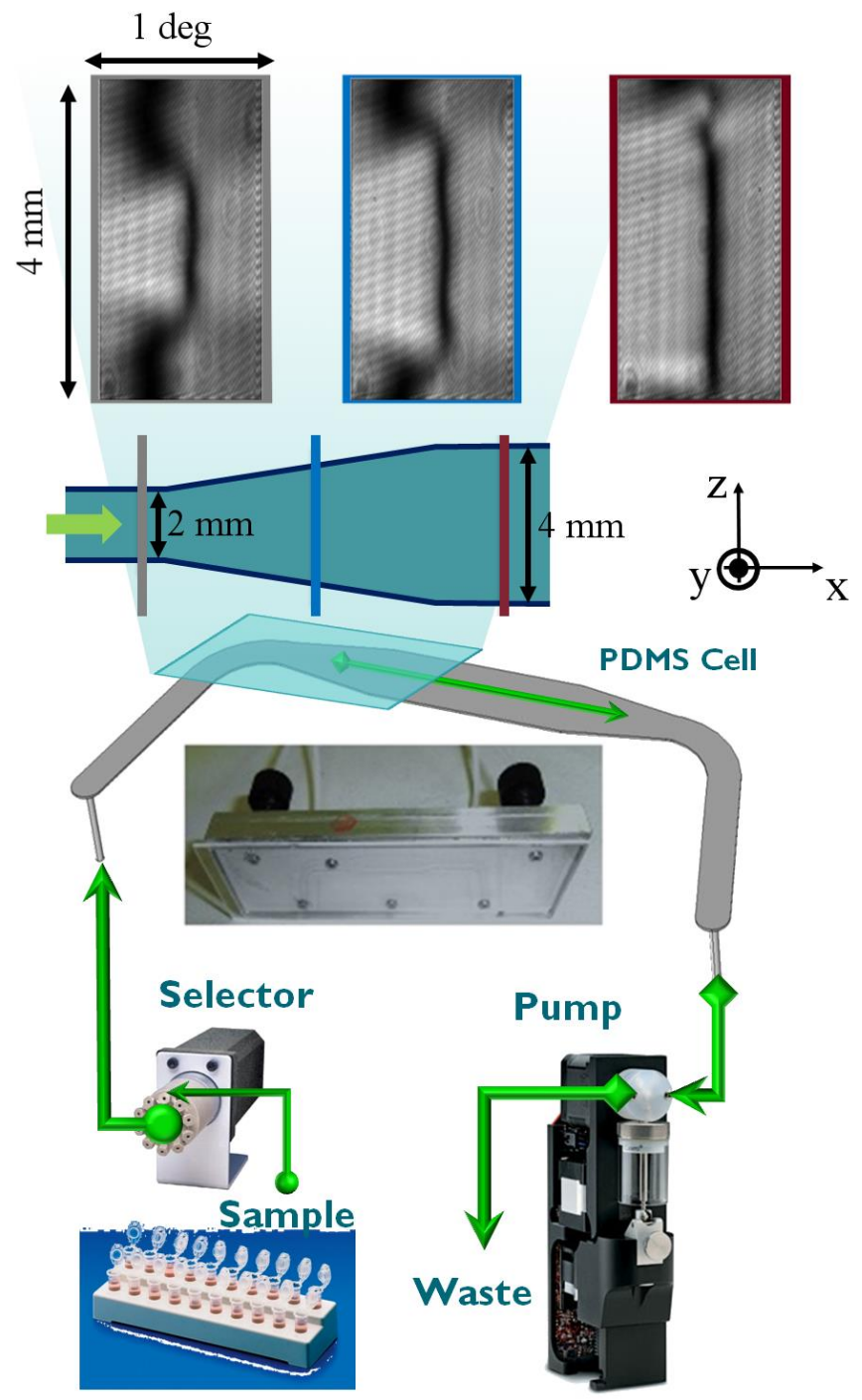

Figure 3: Sketch of the fluids handling system. A photograph of the PDMS cell with tubings is shown in the central inset. At the top we show the CMOS images acquired for different positions of the illumination line along the fluidic channel: across the inlet channel (grey) that is $2 \mathrm{~mm}$ wide, across the inlet channel smothering (blue), and across in the middle part of the channel (red) where the width is maximum (4 mm). The detailed description of the cell is given in Figure S.1.

A. Occhicone et al., Real-time microfluidic monitoring by means of Bloch surface waves at the surface of onedimensional photonic crystals, submitted to Journal of Sensors and Actuators B. 


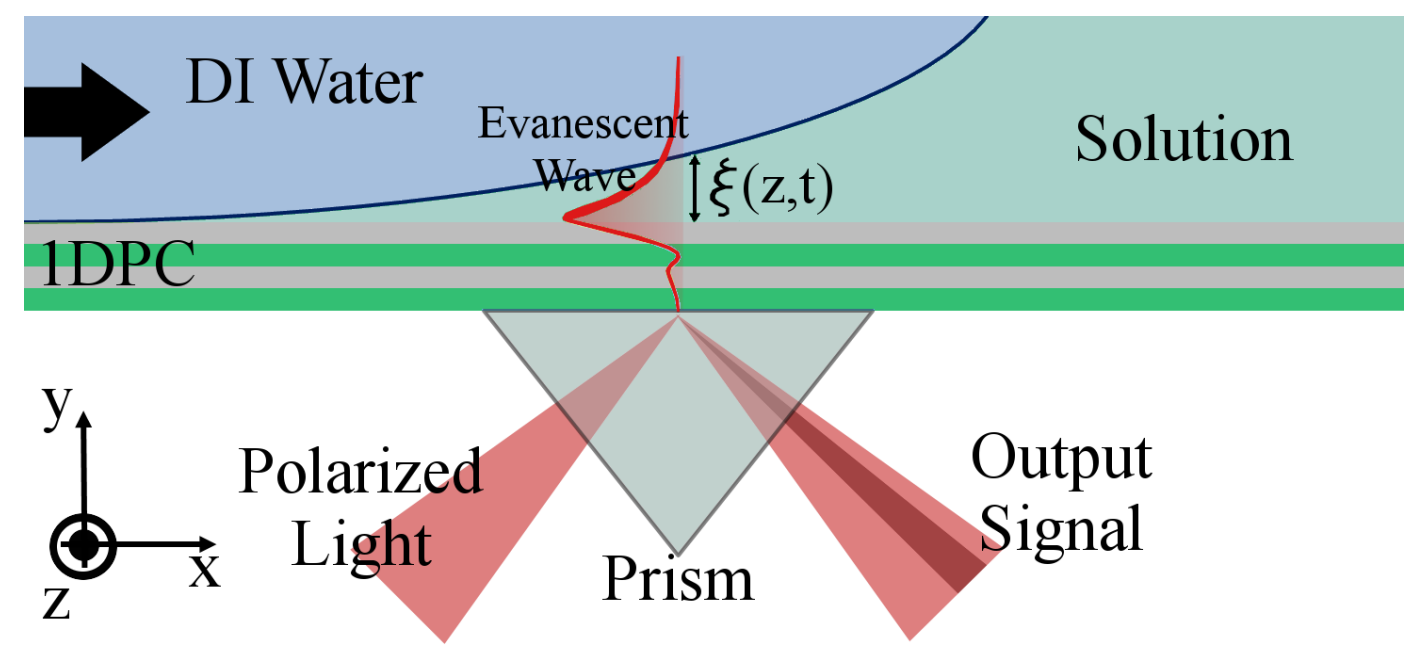

Figure 4: Qualitative representation of the parabolic interface between a solution and the DI water injected in the microfluidic cell. The BSW probes the effective refractive index at the 1DPC surface.

A. Occhicone et al., Real-time microfluidic monitoring by means of Bloch surface waves at the surface of onedimensional photonic crystals, submitted to Journal of Sensors and Actuators B. 
a) $20.95 \mu \mathrm{L} / \mathrm{s}$
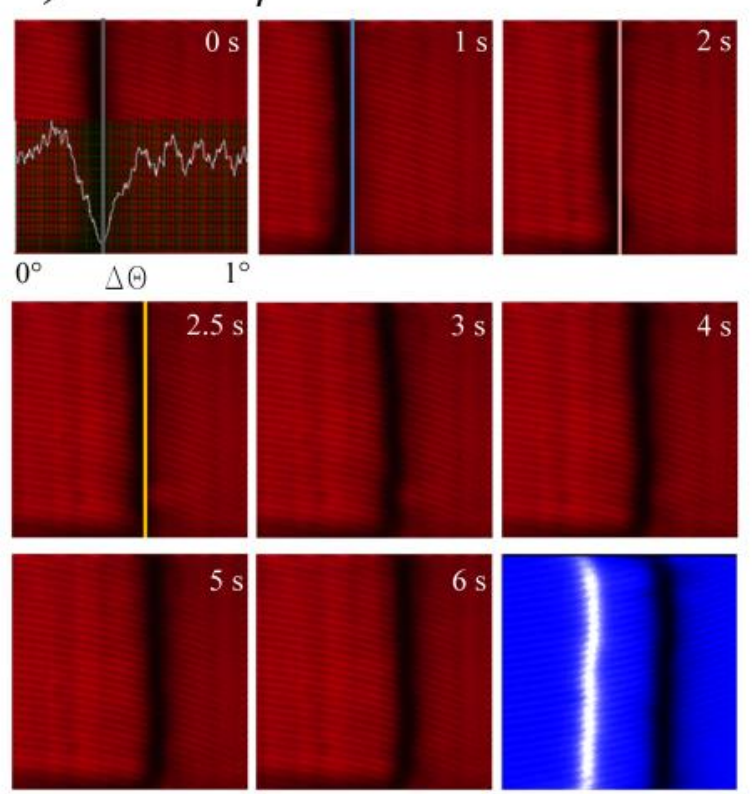

b) $10.52 \mu \mathrm{L} / \mathrm{s}$

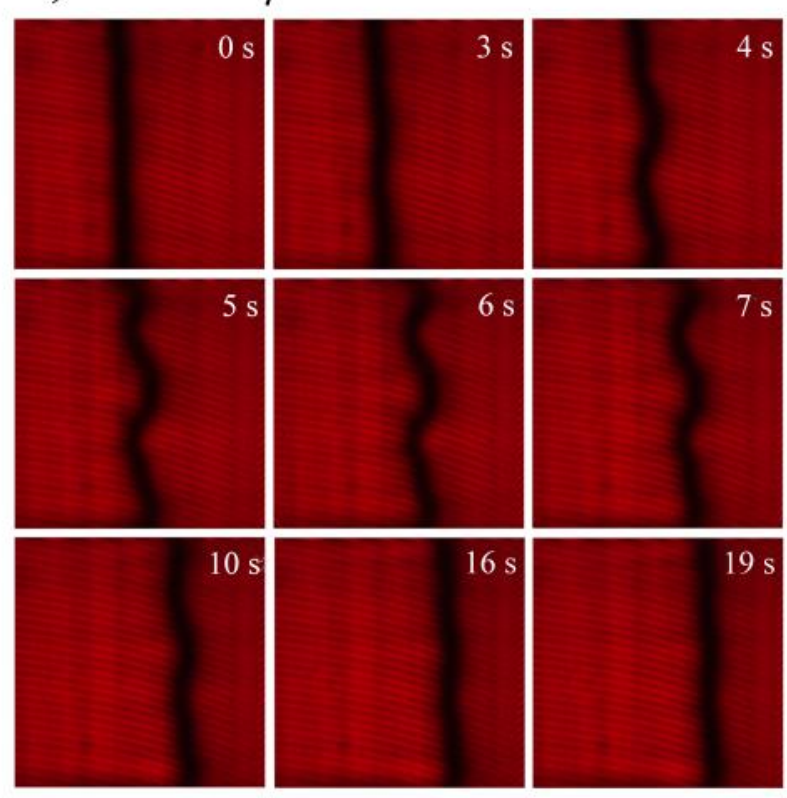

c) $2.699 \mu \mathrm{L} / \mathrm{s}$

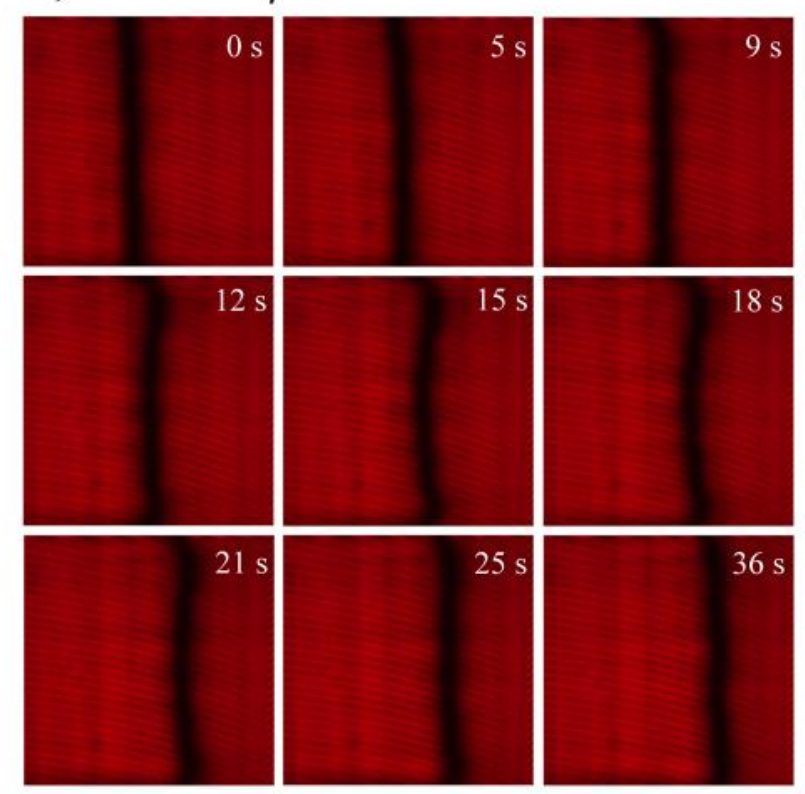

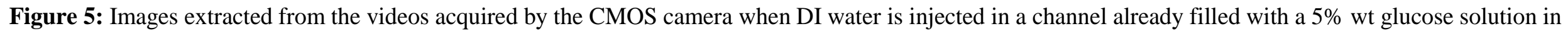
Di water. The images shown here were collected at (a) $\Phi=20.95 \mu \mathrm{L} / \mathrm{s}$, (b) $\Phi=10.52 \mu \mathrm{L} / \mathrm{s}$, and (c) $\Phi=2.699 \mu \mathrm{L} / \mathrm{s}$. In the first frame of (a) we show the profile of the reflected intensity along one of the CMOS camera rows in DI water (row 512). In the last frame of (a) the images obtained at the beginning and at the end of the injection are superimposed to put into e evidence the resonance shift.

A. Occhicone et al., Real-time microfluidic monitoring by means of Bloch surface waves at the surface of one-dimensional photonic crystals, submitted to Journal of Sensors and Actuators $B$. 


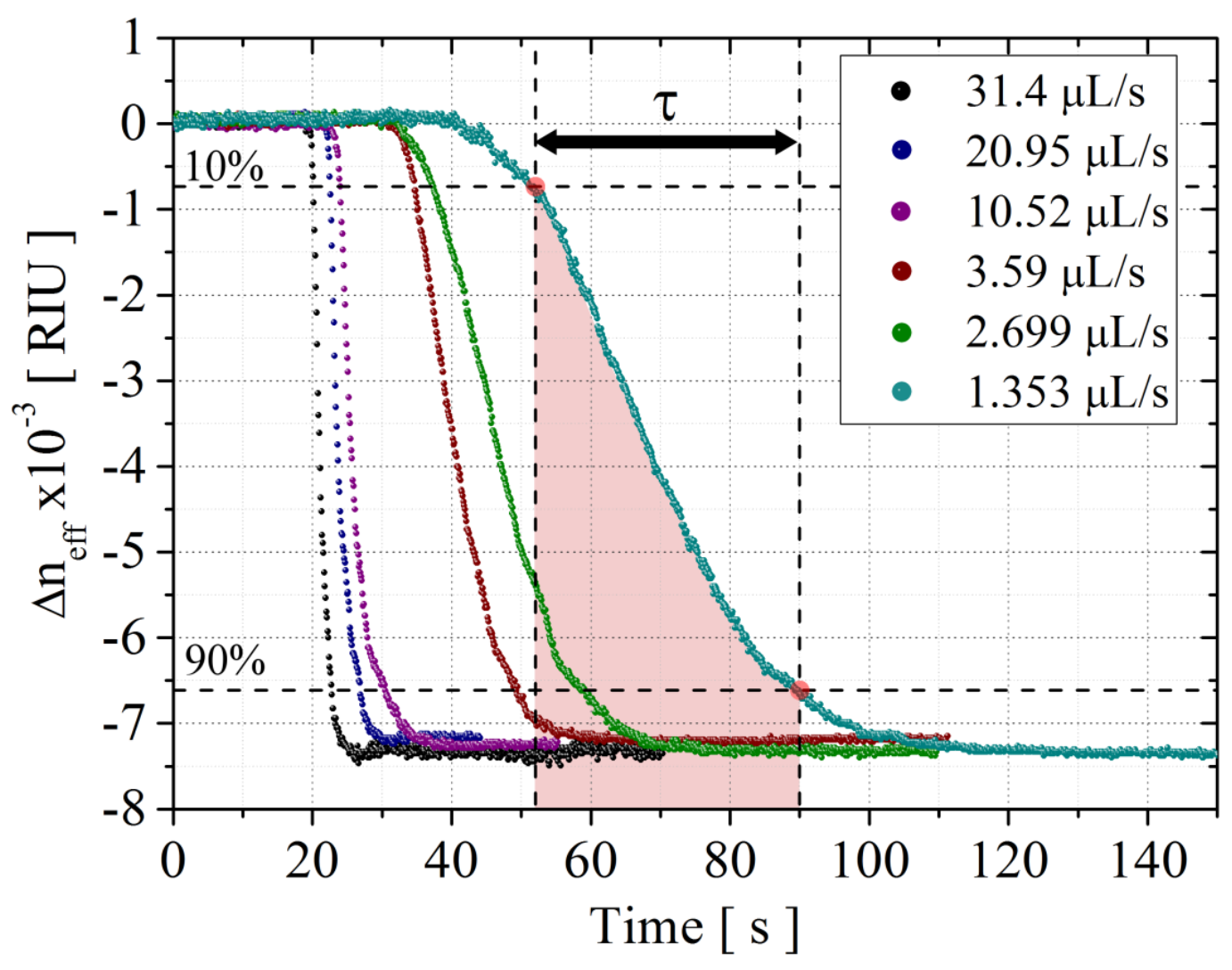

Figure 6: Measured temporal dependence of the effective refractive index change $\Delta \mathrm{n}_{\text {eff }}(\mathrm{t})$ for several different experimental values of $\Phi$. The curves were extracted from measurements similar to those reported in Figure 5 and correspond to the row 512 of the CMOS frames (centre of the channel). The method used to define $\tau$ is shown for the case $\Phi=1.353 \mu \mathrm{L} / \mathrm{s}$.

A. Occhicone et al., Real-time microfluidic monitoring by means of Bloch surface waves at the surface of onedimensional photonic crystals, submitted to Journal of Sensors and Actuators B. 

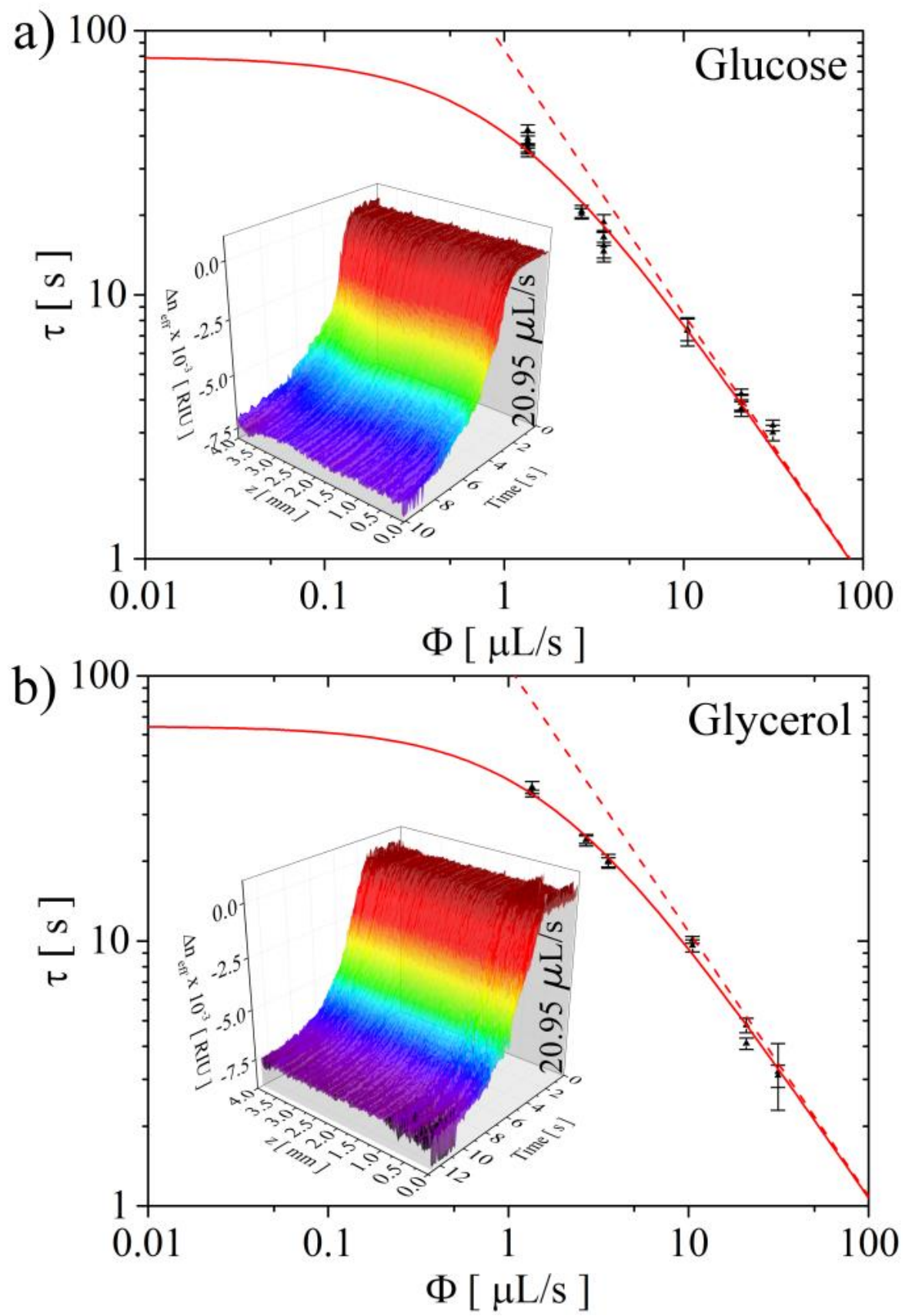

Figure 7: Experimental values of $\tau$ as function of $\Phi$. For a given $\Phi, \tau$ was obtained from the $\Delta \mathrm{n}_{\text {eff }}(\mathrm{z}, \mathrm{t})$ decay surfaces shown in the insets and is the average of the values found across the channel for $\mathrm{z} \in(0.8 \mathrm{~mm}, 3.2$ $\mathrm{mm}$ ) Experiments carried out with 5\% wt solutions in DI water of: (a) glucose (b) glycerol. The red solid line is the interpolation with the Eq. (7) and the dashed line is the linear approximation valid for large $\Phi$ values. The insets show the $\Delta \mathrm{n}_{\text {eff }}(\mathrm{z}, \mathrm{t})$ change across the channel as a function of time in the exemplary case $\Phi=20.95 \mu \mathrm{L} / \mathrm{s}$.

A. Occhicone et al., Real-time microfluidic monitoring by means of Bloch surface waves at the surface of onedimensional photonic crystals, submitted to Journal of Sensors and Actuators B. 


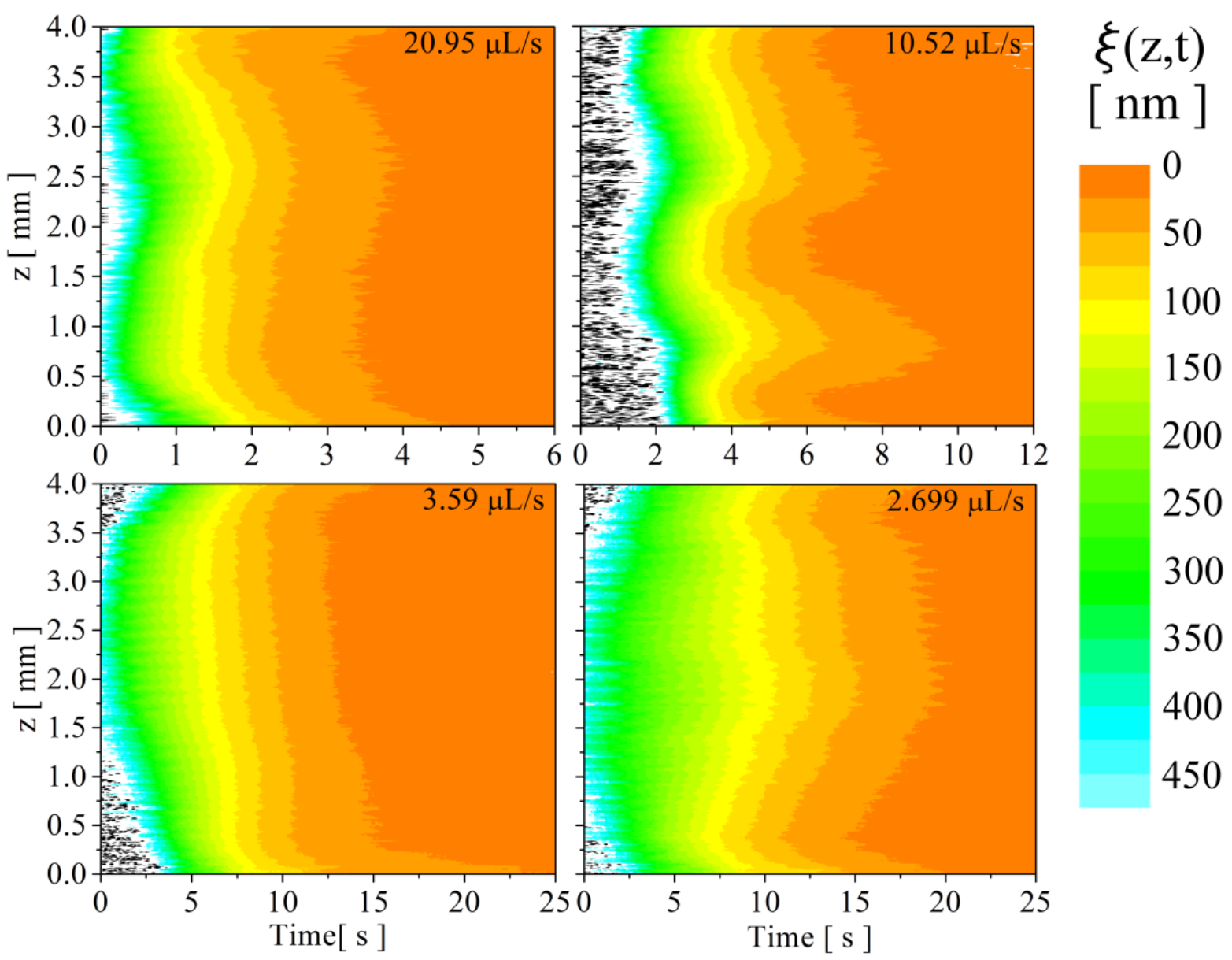

Figure 8: Reconstructed experimental maps of $\xi(\mathrm{z}, \mathrm{t})$, as a function of time and of the position across the fluidic channel, for $\Phi=20.95 \mu \mathrm{L} / \mathrm{s}, 10.52 \mu \mathrm{L} / \mathrm{s}, 3.59 \mu \mathrm{L} / \mathrm{s}$, and $2.699 \mu \mathrm{L} / \mathrm{s}$. The maps put in evidence the temporal evolution of the interface during the injection of DI water when the fluidic cell is filled with a solution 5\% wt glucose in water. The white and black areas correspond to regions where $\xi(\mathrm{z}, \mathrm{t})$ could not be evaluated because the $\xi(\mathrm{z}, \mathrm{t})$ values are larger than the upper limit of the dynamic range of the sensor.

A. Occhicone et al., Real-time microfluidic monitoring by means of Bloch surface waves at the surface of onedimensional photonic crystals, submitted to Journal of Sensors and Actuators B. 